\title{
Transient Ischemic Attack and Hypoventilation 12 Hours After Intra-vitreal Aflibercept Injection
}

Tariq Hamadneh ${ }^{1,2}$, Mohammad Shehadeh ${ }^{3}$, Anas Yasin ${ }^{3}$, Mohammad Akkawi ${ }^{3}$, MeiXia An ${ }^{1}$, Hasan Yamin ${ }^{4}$, Murad Azamtta ${ }^{4}$

1. Ophthalmology Department, The Third Affiliated Hospital of Southern Medical University, Guangzhou, CHN 2. Ophthalmology, California Institute of Behavioral Neurosciences \& Psychology, Fairfield, USA 3. Department of Ophthalmology, Faculty of Medicine and Health Sciences, An-Najah National University Hospital, An-Najah National University, Nablus, PSE 4. Department of Internal Medicine, Faculty of Medicine and Health Sciences, An-Najah National University Hospital, An-Najah National University, Nablus, PSE

Corresponding author: Tariq Hamadneh, tariqqhamadneh@gmail.com

\section{Abstract}

Given the role the vascular endothelial growth factor (VEGF) plays in controlling and preserving the integrity of the vascular endothelium intravitreal administration of anti-VEGF agents may affect the risk of thromboembolic events. This is particularly noticeable in patients who are at risk for atherosclerosis. Here, we present one of the first case reports of transient ischemic attack (TIA) together with hypoventilation secondary to aflibercept injection.

A 63-year-old female suffered TIA together with hypoventilation about 12 hours after the third administration of intravitreal aflibercept, which is a VEGF inhibitor for diabetic macular edema (DME). Upon presentation, she was confused, had right-sided weakness and her respiratory rate was six breaths per minute, all of these resolved within the next 24 hours. The serum tests, cerebrospinal fluid (CSF) analysis, brain imaging, and carotid Doppler ultrasound were unremarkable. An ophthalmic exam revealed signs of bilateral proliferative diabetic retinopathy with left macular edema. Detailed reports of similar cases are lacking in the literature. Hypoventilation and thromboembolic could be possible side effects of aflibercept that necessitate more investigation.

Received 01/24/2021 Review began 02/01/2021 Review ended 02/12/2021 Published 02/22/2021

\section{() Copyright 2021}

Hamadneh et al. This is an open access article distributed under the terms of the Creative Commons Attribution License CC-BY 4.0., which permits unrestricted use, distribution, and reproduction in any medium, provided the original author and source are credited.
Categories: Internal Medicine, Ophthalmology

Keywords: aflibercept, transient ischemic attack, hypoventilation, vegf inhibitors

\section{Introduction}

Vascular endothelial growth factor (VEGF), also known as vasculotropin, is a dimeric, heparin-binding, polypeptide mitogen with four isoforms produced from alternative mRNA splicing [1]. In actively proliferating vascular tissue, the VEGF gene is widely expressed, and VEGF is considered a critical mediator of physiological and pathological angiogenesis [1]. It activates endothelial cells, promotes cell proliferation and migration, and increases vascular permeability [2]. Angiogenesis inhibitors, for example anti-VEGF inhibitors, have been classically used to treat neoplasia. Later, its use was expanded to target retinal angiogenesis related to several retinal pathologies such as age-related macular degeneration (AMD) and diabetic macular edema (DME) [3].

Three commonly used intravitreal VEGF inhibitors-aflibercept (Eylea ${ }^{\circledR}$, Regeneron Pharmaceuticals), bevacizumab (Avastin ${ }^{\circledR}$, Genentech), and ranibizumab (Lucentis ${ }^{\circledR}$, Genentech)-are effective as angiogenesis inhibitors and relatively safe for the treatment of DME, but only aflibercept and ranibizumab are approved by the Food and Drug Administration (FDA) for this indication [1]. Bevacizumab, which is not approved by the FDA for any ocular indication, is widely used for off-label treatment of DME [4-5].

Considering the role that VEGF plays in the regulation of vascular endothelium and maintaining its integrity, anti-VEGF agents may have the potential risk of thromboembolic events, even with intravitreal administration. This is particularly evident in patients at risk of atherosclerosis [6-9].

Here, we report a case of TIA and hypoventilation developed within 12 hours of aflibercept administration for DME. Informed consent was obtained from the patient for this study.

\section{Case Presentation}

A 63-year-old female patient was admitted to An-Najah National University Hospital (NNUH) intensive care unit (ICU) for a decreased level of consciousness and hypoventilation. Her past medical history was significant for hypertension and type II diabetes mellitus complicated by proliferative retinopathy and nephropathy. The patient's symtoms started one day before presentation when she had sudden onset rightsided weakness with aphasia and vomiting of normal gastric content with up rolling eyes and saliva drooling 
followed by a decreased level of consciousness and shortness of breath. By further questioning, her family denied a history of previous similar episodes. There was no history of fever, photophobia, neck stiffness, falling, or any psychosocial problems. She had not traveled or had any contact with sick people.

She received six bevacizumab (Avastin ${ }^{\circledR} 1.25 \mathrm{mg}$ in $0.05 \mathrm{ml}$ per injection) injections during the last two years period for optical coherence tomography (OCT) confirmed DME. Then, she was prescribed aflibercept (Eylea ${ }^{\circledR} 2 \mathrm{mg}$ in $0.05 \mathrm{ml}$ per injection) [5]. Two injections were given over the past four months with no complications. However, she suffered her current symptoms only 12 hours after the third injection of aflibercept.

On presentation, the patient was confused; her vital signs were significant for hypopnea, with a respiratory rate (RR) of six breaths per minute, and hypoxemia with a normal alveolar-arterial gradient (A-a gradient). Her physical exam showed mild weakness in the right side of the body; the rest of her neurological and systemic examination was unremarkable. The patient was initially diagnosed as having a transient ischemic attack with hypoventilation and further investigations to look for underlying pathology were ordered.

Routine chemistry was unremarkable except for 2.7 gm of proteinuria. Imaging studies including chest x-ray, brain computed tomography (CT), brain magnetic resonance imaging (MRI), brain magnetic resonance angiogram (MRA), echocardiogram, and carotid Doppler ultrasound were all within normal limits. CSF analysis was also normal with no signs of pleocytosis, xanthochromia, or red blood cells. An ophthalmic exam was performed and revealed bilateral proliferative diabetic retinopathy with left macular edema. Optic discs were within normal limits.

She received non-invasive ventilation in the ICU, and she improved over 24 hours with complete recovery of power and respiration.

\section{Discussion}

Aflibercept is a recombinant fusion protein consisting of the extracellular domain of VEGF and the Fc region of human immunoglobulin G1 (IgG1) [9]. In contrast to bevacizumab and ranibizumab which bind only VEGF-A, aflibercept also binds to VEGF-B and the placental growth factor, which are also important angiogenic factors [3,10]. Aflibercept was approved by the FDA in 2011 for the treatment of AMD, and in 2014 for the treatment of DME [11].

In the context of current evidence of a reduction in circulating VEGF levels after intravitreal anti-VEGF injection, concerns regarding their safety and systemic adverse effects are understandable [12]. Historically, the intravenous use of the bevacizumab in the management of various cancers had been associated with higher rates of cerebrovascular events [13-14]. Given the fact of systemic absorption, the occurrence of these adverse effects after intravitreal use is not surprising. For instance, a 2-year data from the IVAN trial showed a statistically significant higher risk of cardiovascular events with bevacizumab than with ranibizumab [15]. Other studies linked the use of aflibercept with an increase in stroke incidence especially in those patients already with high baseline risk [16].

Generally, the reduction in plasma free-VEGF levels correlates with elevated levels of circulating anti-VEGF agents, with the reduction in free-VEGF levels greatest with aflibercept and least with ranibizumab [17]. This along with the wider spectrum of angiogenic factors inhibition may explain why aflibercept can be associated with higher cerebrovascular accidents. This issue is further amplified in the elderly, patients with diabetes, and those with recent stroke who may be at increased risk at the time of administration $[12,16]$.

On a molecular level, binding of aflibercept to VEGF-A not only decreases VEGF concentration but also acts as a potent pro-inflammatory signal for increased expression of atherosclerosis-associated inflammatory mediators and cytokines which is in part mediated by Toll-like receptor 4 activation on the endothelial cell surface [18].

Although a limited number of case reports described thromboembolic event post aflibercept use, this is the first case reporting a possible association between aflibercept, TIA, and hypoventilation given the negative result of all investigations [19-20]. Moreover, apart from this potential link between aflibercept and TIA, a synergetic effect of aflibercept to the atherosclerotic consequences of age, diabetes mellitus and hypertension may have contributed to the occurrence of the ischemic event in our patient.

\section{Conclusions}

VEGF inhibitors are commonly used as angiogenesis inhibitors for many ocular diseases. Because VEGF plays an important role in regulating and preserving the integrity of the endothelium, VEGF inhibitors can contribute to the development of thromboembolic events especially in patients who are at risk for atherosclerotic vascular diseases. Given the potential of systemic absorption after intravitreal VEGF inhibitors administration, systemic adverse effects of these agents are understandable. However, the exact association between VEGF inhibitors use and the risk of thromboembolism is still not clear and detailed case 
reports regarding this issue are lacking. We recommend further studies to investigate the systemic adverse effects of VEGF inhibitors as intraocular injections, especially the possibility of thromboembolic events.

\section{Additional Information \\ Disclosures}

Human subjects: Consent was obtained or waived by all participants in this study. Conflicts of interest: In compliance with the ICMJE uniform disclosure form, all authors declare the following: Payment/services info: All authors have declared that no financial support was received from any organization for the submitted work. Financial relationships: All authors have declared that they have no financial relationships at present or within the previous three years with any organizations that might have an interest in the submitted work. Other relationships: All authors have declared that there are no other relationships or activities that could appear to have influenced the submitted work.

\section{Acknowledgements}

We thank An-Najah National University Hospital for granting us the privilege to conduct this case report.

\section{References}

1. 2019-2020 Basic and Clinical Science Course, Section 2: Fundamentals and Principles of Ophthalmology . Vikram S. Brar (ed): American Academy of Ophthalmology, San Francisco, United States; 2019. 12:475.

2. Crawford Y, Ferrara N: VEGF inhibition: insights from preclinical and clinical studies . Cell Tissue Res. 2009, 335:261-9. 10.1007/s00441-008-0675-8

3. Ferrara N, Damico L, Shams N, Lowman H, Kim R: Development of ranibizumab, an anti-vascular endothelial growth factor antigen binding fragment, as therapy for neovascular age-related macular degeneration. Retina. 2006, 26:859-70. 10.1097/01.iae.0000242842.14624.e7

4. Korobelnik JF, Do DV, Schmidt-Erfurth U, et al.: Intravitreal aflibercept for diabetic macular edema . Ophthalmology. 2014, 121:2247-54. 10.1016/j.ophtha.2014.05.006

5. Diabetic Retinopathy Clinical Research Network, Wells JA, Glassman AR, et al.: Aflibercept, bevacizumab, or ranibizumab for diabetic macular edema. N Engl J Med. 2015, 372:1193-203. 10.1056/NEJMoa1414264

6. Schmidt-Erfurth U: Clinical safety of ranibizumab in age-related macular degeneration . Expert Opin Drug Saf. 2010, 9:149-65. 10.1517/14740330903418422

7. Lim LS, Cheung CMG, Mitchell P, Wong TY: Emerging evidence concerning systemic safety of anti-VEGF agents--should ophthalmologists be concerned?. Am J Ophthalmol. 2011, 152:329-331. 10.1016/j.ajo.2011.05.040

8. Schmucker C, Loke YK, Ehlken C, Agostini HT, Hansen LL, Antes G, Lelgemann M: Intravitreal bevacizumab (Avastin) versus ranibizumab (Lucentis) for the treatment of age-related macular degeneration: a safety review. Br J Ophthalmol. 2011, 95:308-17. 10.1136/bjo.2009.178574

9. Papadopoulos N, Martin J, Ruan Q, et al.: Binding and neutralization of vascular endothelial growth factor (VEGF) and related ligands by VEGF Trap, ranibizumab and bevacizumab. Angiogenesis. 2012, 15:171-85. 10.1007/s10456-011-9249-6

10. Ferrara N, Hillan KJ, Gerber HP, Novotny W: Discovery and development of bevacizumab, an anti-VEGF antibody for treating cancer. Nat Rev Drug Discov. 2004, 3:391-400. 10.1038/nrd1381

11. Verner-Cole EA, Davis SJ, Lauer AK: Aflibercept for the treatment of neovascular age-related macular degeneration. Drugs Today (Barc). 2012, 48:317-29. 10.1358/dot.2012.48.5.1805931

12. Avery RL, Castellarin AA, Steinle NC, et al.: Systemic pharmacokinetics following intravitreal injections of ranibizumab, bevacizumab or aflibercept in patients with neovascular AMD. Br J Ophthalmol. 2014, 98:1636-41. 10.1136/bjophthalmol-2014-305252

13. Ranpura V, Hapani S, Chuang J, Wu S: Risk of cardiac ischemia and arterial thromboembolic events with the angiogenesis inhibitor bevacizumab in cancer patients: a meta-analysis of randomized controlled trials. Acta Oncol. 2010, 49:287-97. 10.3109/02841860903524396

14. Scappaticci FA, Skillings JR, Holden SN, et al.: Arterial thromboembolic events in patients with metastatic carcinoma treated with chemotherapy and bevacizumab. J Natl Cancer Inst. 2007, 99:1232-9. 10.1093/jnci/djm086

15. Chakravarthy U, Harding SP, Rogers CA, et al.: Alternative treatments to inhibit VEGF in age-related choroidal neovascularisation: 2-year findings of the IVAN randomised controlled trial. Lancet. 2013, 382:1258-67. 10.1016/S0140-6736(13)61501-9

16. Avery RL: What is the evidence for systemic effects of intravitreal anti-VEGF agents, and should we be concerned?. Br J Ophthalmol. 2014, 98:i7-10. 10.1136/bjophthalmol-2013-303844

17. Avery RL, Castellarin AA, Steinle NC, et al.: Systemic pharmacokinetics and pharmacodynamics of intravitreal aflibercept, bevacizumab, and ranibizumab . Retina. 2017, 37:1847-1858. 10.1097/IAE.0000000000001493

18. Arnott C, Punnia-Moorthy G, Tan J, Sadeghipour S, Bursill C, Patel S: The vascular endothelial growth factor inhibitors ranibizumab and aflibercept markedly increase expression of atherosclerosis-associated inflammatory mediators on vascular endothelial cells. PLoS One. 2016, 11:e0150688. 10.1371/journal.pone.0150688

19. Thorel J, Civade E, Quintyn JC, Cestac P, Montastruc JL, Bagheri H: Ischaemic stroke after exposure to aflibercept: interaction with vitamin K antagonist and/or direct pharmacodynamic effect?. J Clin Pharm Ther. 2015, 40:477-9. 10.1111/jcpt.12278

20. Mizutani H, Inatomi Y, Singu T, Nakajima M, Yonehara T, Ando Y: [Embolic stroke immediately after initial administration of intravitreal aflibercept]. Rinsho Shinkeigaku. 2018, 58:314-319. 


\section{Cureus}

10.5692/clinicalneurol.cn-001162 